\title{
Correlations between pulse oximetry and peak expiratory flow in acute asthma
}

\section{Ribeiro de Andrade ${ }^{1}$, \\ M.C. Duarte ${ }^{2}$ and P. Camargos ${ }^{1}$}

\author{
${ }^{1}$ Departamento de Pediatria, Faculdade de Medicina, \\ Universidade Federal de Minas Gerais, Belo Horizonte, MG, Brasil \\ ${ }^{2}$ Departamento de Saúde Materna e Infantil, Faculdade de Medicina, \\ Universidade Federal de Juiz de Fora, Juiz de Fora, MG, Brasil
}

\section{Correspondence}

P. Camargos

Departamento de Pediatria

Faculdade de Medicina, UFMG

Av. Alfredo Balena, 190, Sala 4061

30130-100 Belo Horizonte, MG

Brasil

Fax: +55-31-3248-9664

E-mail: pcamargs@medicina.ufmg.br

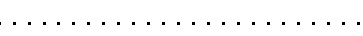

Received March 28, 2006

Accepted January 15, 2007

\section{Abstract}

Few studies are available concerning correlations between pulse oximetry and peak expiratory flow in children and adolescents with acute asthma. Although the Global Initiative for Asthma states that measurements of lung function and oximetry are critical for the assessment of patients, it is not clear if both methods should necessarily be included in their evaluation. Since there is a significant difference in cost between pulse oximetry equipment and peak expiratory flow devices, we determined whether clinical findings and peak expiratory flow measurements are sufficient to determine the severity of acute asthma. The present prospective observational study was carried out to determine if there is correlation between pulse oximetry and peak expiratory flow determination in 196 patients with acute asthma aged 4 to 15 years diagnosed according to the Global Initiative for Asthma criteria. Patients experiencing their first or second wheezing episode, with fever, related acute or chronic diseases, and unable to perform the peak expiratory flow maneuver were excluded. Measurements of peak expiratory flow and pulse oximetry were performed at admission and after $15 \mathrm{~min}$ of each inhaled salbutamol cycle. Correlations obtained by linear regression using the Pearson correlation coefficients $(\mathrm{r})$ were $0.41(\mathrm{P}<0.0001), 0.53(\mathrm{P}<0.0001), 0.51(\mathrm{P}$ $<0.0001)$, and $0.61(\mathrm{P}<0.0001)$ at admission and after the first, second and third cycles of salbutamol, respectively. These correlations showed that one measure cannot substitute the other (Pearson's coefficient $<0.7$ ), probably because they evaluate different aspects in the airways, suggesting that peak expiratory flow should not be used alone in the assessment of acute asthma in children and adolescents.

\section{Introduction}

Clinical findings are usually insufficient to properly determine the severity of acute asthma (1-9). Significant changes in lung function may be present despite the lack of clinical manifestations $(1,2,6)$, as first re-
Key words

- Pulse oximetry

- Peak expiratory flow
- Acute asthma ported in the 1970's (1) and confirmed by subsequent studies (2-9). The severity of acute asthma is classified as mild, moderate, severe, and very severe based on clinical and functional criteria (10), the most widely used being pulse oximetry $\left(\mathrm{SpO}_{2}\right)$ and peak expiratory flow (PEF). 
Few reports are available about possible correlations between these two methods in children and adolescents. Although the Global Initiative for Asthma (GINA) states that measurements of lung function and oximetry are critical for patient assessment (10), it is not clear whether both methods should be included in the evaluation of acute asthma, since no critical comparison of the two measurements has been done.

Since there is a significant difference in costs between $\mathrm{SpO}_{2}$ equipment and PEF devices, with $\mathrm{SpO}_{2}$ being about 20 times more expensive, it seems reasonable to determine whether clinical findings and PEF measurements are sufficient to assess the severity of acute asthma. Thus, the objective of the present study was to determine the correlation between $\mathrm{SpO}_{2}$ and PEF in children and adolescents with acute asthma in order to determine whether $\mathrm{SpO}_{2}$, the more expensive procedure, could be avoided in the evaluation of exacerbations. The answer may be particularly relevant in low-income countries, where the two measurements are not performed routinely in emergency departments.

\section{Material and Methods}

In this prospective cohort study, 196 consecutive patients with acute asthma were evaluated. The patients were admitted to an emergency room where they were treated with standard doses of inhaled salbutamol.

\section{Inclusion and exclusion criteria}

Since most asthma exacerbations are classified as mild or moderate (10), patients with this level of severity, aged 4 to 15 years, and with PEF between 50 and $80 \%$ of the predicted value at admission were studied. Patients experiencing their first or second wheezing episode, patients with axillary temperature of $38^{\circ} \mathrm{C}$ or higher, with related acute or chronic diseases, and patients unable to perform the PEF maneuver were excluded.

Each patient was included only once in the study protocol.

\section{Pulse oximetry and peak expiratory flow}

$\mathrm{SpO}_{2}$ was measured with a Palco oximeter model 30 (Palco Laboratories, Inc., Santa Cruz, CA, USA) at admission to the emergency room and after $15 \mathrm{~min}$ of each bronchodilator cycle. The sensor was adapted to the patient's left thumb. Single point values were recorded in order to ensure a highquality pulse signal and no movement artifacts.

PEF was measured with a peak flow meter (Mini Wright Peak Flow Meter; Clement Clarke International Ltd., Harlow, Essex, England) on a non-linear scale ranging from 30 to $400 \mathrm{~L} / \mathrm{min}$. The best of three standing PEF values was recorded and the results were compared to the reference values described by Godfrey et al. (11).

Measurements were performed at admission and after 15 min of each inhaled salbutamol cycle. PEF and $\mathrm{SpO}_{2}$ were measured by an observer unaware of the study aims.

\section{Acute asthma treatment}

Patients received up to three cycles of salbutamol delivered either by an oxygendriven nebulizer or by a pressurized metered-dose inhaler attached to a spacer. Those treated with the oxygen-driven nebulizer received $0.15 \mathrm{mg} \mathrm{kg}^{-1}$ dose $^{-1}$ (minimum: 1.25 $\mathrm{mg} / \mathrm{dose}$; maximum: $5 \mathrm{mg}$ /dose). The drug was diluted in $0.9 \%$ sodium chloride to a final volume of $4 \mathrm{~mL}$. Nebulization was performed up to $15 \mathrm{~min}$, with the device connected to the oxygen source and at a flow of $6 \mathrm{~L} / \mathrm{min}$. Those treated with the pressurized metered-dose inhaler attached to a valved spacer received 5 puffs $(500 \mu \mathrm{g})$ in each treatment cycle.

Patients were assessed clinically and functionally, i.e., by $\mathrm{PEF}$ and $\mathrm{SpO}_{2}$ measure- 
ments, approximately 15 min after each salbutamol cycle. Emergency room discharge was conditioned to a satisfactory therapeutic response represented by improvement of one or more of the following factors: cough, wheezing, shortness of breath, decreased respiratory frequency, respiratory effort, and increased $\mathrm{SpO}_{2}$ and PEF values.

\section{Statistical analysis}

Sample size. Since in three previous studies the correlation coefficient $(\mathrm{r}$ ) ranged from -0.16 to 0.43 for $\mathrm{SpO}_{2}$ and PEF (12-14), an intermediate value was taken $(r=0.25)$ for the calculation of sample size. Assuming an alpha error of 0.05 and a beta error of 0.10 (power equal to 90\%), a sample size of 164 patients was required (15).

Analysis. Descriptive statistics were calculated on the basis of frequency distribution and by the Student $t$-test. Linear regression analysis was used to determine the relationship between $\mathrm{SpO}_{2}$ measurements and predicted PEF values at admission and after each bronchodilator cycle. A $\mathrm{P}$ value $<0.05$ was considered significant.

\section{Ethics}

The study protocol was approved by the Research Ethics Committee of the Federal University of Minas Gerais and the parents or persons responsible for the children gave written informed consent.

\section{Results}

Table 1 presents the demographic and clinical characteristics of the 196 patients studied. Boys predominated in the study population with a 2:1 ratio. Age ranged from 4 to 15 years (mean \pm SD: $8.7 \pm 2.19$ years). Regarding asthma characteristics, the first exacerbation occurred before two years of age in $71.4 \%$ of the patients and only approximately $50 \%$ of the subjects were asymp- tomatic between exacerbations and had acute attacks at 8- to 30-day intervals. Previous asthma hospitalization and use of antiasthmatic medicines was reported by about one third of the patients. Bronchodilators and/or antibiotics had been used within $24 \mathrm{~h}$ preceding emergency room admission by 86 patients, although none within $8 \mathrm{~h}$ or less.

Table 2 shows $\mathrm{SpO}_{2}$ and PEF values at admission and after three cycles of bronchodilator treatment. The mean $\pm \mathrm{SD} \mathrm{SpO}_{2}$ for the 196 patients was $93.6 \pm 2.90$ and 95.0 $\pm 2.70 \%$ at admission and after the first salbutamol cycle, respectively $(\mathrm{P}<0.001)$.

Table 1. Characteristics of the study population.

\begin{tabular}{lr}
\hline Characteristics & $N(\%)$ \\
\hline Gender & \\
Male & $132(67 \%)$ \\
Female & $64(33 \%)$ \\
Age group & \\
4 to 5 years & $18(9 \%)$ \\
6 to 9 years & $114(58 \%)$ \\
10 to 12 years & $62(32 \%)$ \\
13 to 15 years & $2(1 \%)$ \\
& \\
Age at first exacerbation & \\
$<2$ years & $140(71 \%)$ \\
$>2$ years & $56(29 \%)$ \\
Between exacerbation periods & \\
Asymptomatic & $102(52 \%)$ \\
Symptomatic & $94(48 \%)$ \\
Days between exacerbations & \\
7 days & $40(21 \%)$ \\
8 to 30 days & $103(52 \%)$ \\
$>30$ days & $53(27 \%)$
\end{tabular}

Number of hospitalizations due to acute asthma None $127(65 \%)$

$122(11 \%)$

2 or more $\quad 47(24 \%)$

Previous use of asthma-preventing medications Yes $62(32 \%)$ No $134(68 \%)$

Use of bronchodilators and/or anti-inflammatory drugs in the last $24 \mathrm{~h}$

Yes $86(44 \%)$

No $110(56 \%)$

Data are reported as number of patients with percent in parentheses. 
The mean PEF was $61.8 \pm 10.60$ at admission and $80.2 \pm 13.09 \%$ after the first treatment cycle $(\mathrm{P}<0.001)$. In the present study, only $4 \%$ of the subjects showed a decrease in $\mathrm{SpO}_{2}$ after the first salbutamol cycle.

All correlations between $\mathrm{SpO}_{2}$ and $\mathrm{PEF}$ (\% predicted value) determined by the Pear-

Table 2. Pulse oximetry and peak expiratory flow reported as percent predicted values at admission and after each of the three salbutamol cycles.

\begin{tabular}{|c|c|}
\hline & Mean \pm SD \\
\hline \multicolumn{2}{|c|}{ Admission ( $N=196)$} \\
\hline $\mathrm{SpO}_{2}$ & $93.6 \pm 2.9$ \\
\hline PEF & $61.8 \pm 10.6$ \\
\hline \multicolumn{2}{|c|}{ After 1st cycle $(N=196)$} \\
\hline $\mathrm{SpO}_{2}$ & $95.0 \pm 2.7$ \\
\hline PEF & $80.2 \pm 13.1$ \\
\hline \multicolumn{2}{|c|}{ After 2 nd cycle $(N=123)$} \\
\hline $\mathrm{SpO}_{2}$ & $94.8 \pm 2.7$ \\
\hline PEF & $81.0 \pm 9.9$ \\
\hline \multicolumn{2}{|c|}{ After 3rd cycle $(\mathrm{N}=72)$} \\
\hline $\mathrm{SpO}_{2}$ & $95.2 \pm 2.4$ \\
\hline PEF & $82.1 \pm 7.6$ \\
\hline
\end{tabular}

$\mathrm{SpO}_{2}=$ pulse oximetry; $\mathrm{PEF}=$ peak expiratory flow. son coefficient were statistically significant during any phase of treatment $(\mathrm{P}<0.001)$ but to a moderate extent, with " $r$ " values of $0.41,0.53,0.51$, and 0.61 at admission and after the first, second and third salbutamol cycle, respectively. Regarding the determination coefficient $\left(\mathrm{R}^{2}\right)$, the values obtained were statistically significant $(\mathrm{P}<0.0001)$ at admission and after each salbutamol cycle. Values found at admission and after the first, second, and third cycles were $0.17,0.29$, 0.26 , and 0.37 , respectively, as shown in Figure 1.

Figure 1 shows the four scattergrams corresponding to each assessment. The Pearson coefficients with their respective P values and 95\% confidence intervals are also shown.

A positive correlation can be observed between the two measurements at admission and after each salbutamol cycle. Since only patients suffering from mild to moderate acute asthma were admitted to the study, most points observed in the scattergrams were concentrated in values ranging from 92 to $98 \%$ and from 70 to $95 \%$ for $\mathrm{SpO}_{2}$ and PEF, respectively. However, in $25 \%$ of the
Figure 1. Scattergrams plotting pulse oximetry and peak expiratory flow. A, At admission ( $\mathrm{N}=$ 196). $B$, First cycle $(\mathrm{N}=196)$. $C$, Second cycle $(\mathrm{N}=123)$. $D$, Third cycle $(\mathrm{N}=72)$. $\mathrm{PEF}=$ peak expiratory flow; $\mathrm{SpO}_{2}=$ pulse oximetry; $r=$ Pearson correlation coefficients; $\mathrm{Cl}=$ confidence interval.

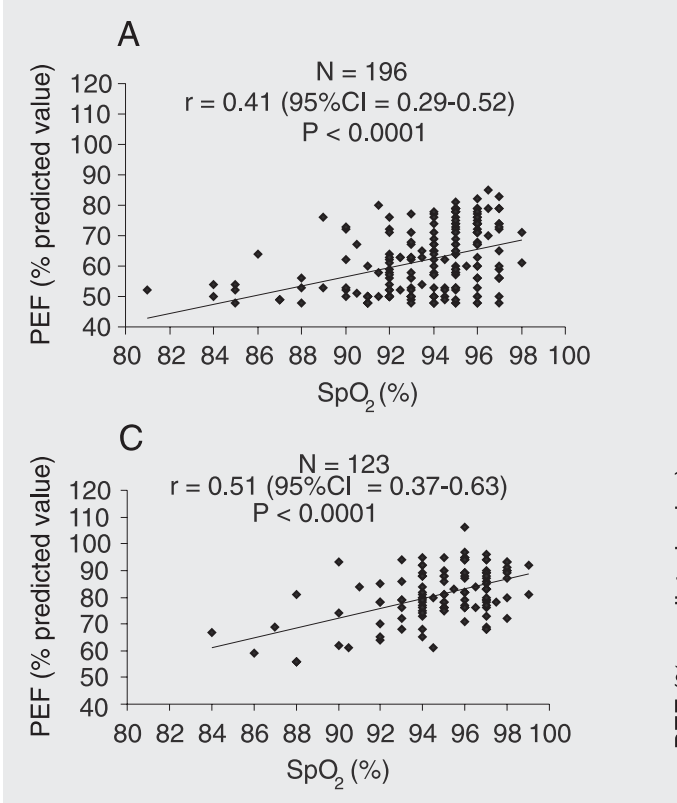

\section{B}

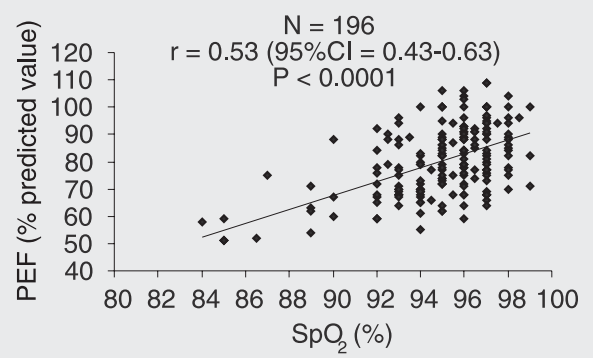

D

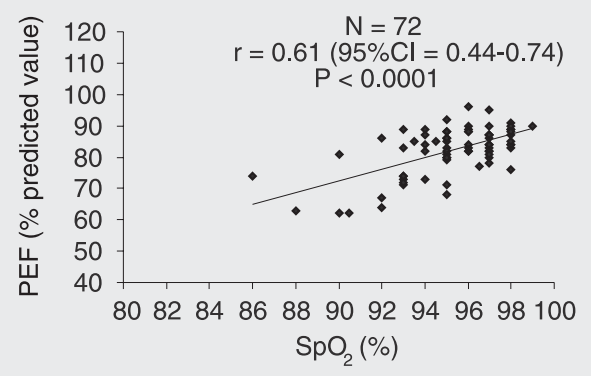


subjects discrepancies were observed for both $\mathrm{SpO}_{2}<95 \%$ and PEF $\geq 80 \%$ of the predicted value and vice versa. Some patients with PEF values above $70 \%$ of the predicted value showed low $\mathrm{SpO}_{2}$ values. Furthermore, $6 \%$ of the patients presented $\mathrm{SpO}_{2}<90 \%$ and $\mathrm{PEF} \geq 60 \%$ of the predicted value.

\section{Discussion}

The correlations between $\mathrm{SpO}_{2}$ and PEF values obtained in the present study were statistically significant in every salbutamol cycle. There are few reports in the literature concerning the use of both measurements in acute asthma, although GINA and most Brazilian asthma guidelines recommend both for the management of patients with acute asthma in emergency rooms.

Only three studies have measured the correlation between $\mathrm{SpO}_{2}$ and PEF in acute asthma in childhood and adolescence (1214). All of them have different methodologies compared with the present study and in only one study the investigators performed sequential assessments of $\mathrm{SpO} 2$ and $\mathrm{PEF}$ (13) as we did. Kano and Nishima (13) detected a correlation in only 52 patients aged 6 to 16 years before and after one bronchodilator cycle, obtaining $\mathrm{r}=0.47$ and 0.52 , respectively $(\mathrm{P}<0.0001)$. Their limited number of subjects should be taken into consideration. Yamamoto et al. (14), in a study of 632 patients aged 5 to 20 years with acute wheezing episodes, obtained $r=0.41$ at admission, a value similar to that obtained in the present study $(\mathrm{P}<0.0001)$. However, these investigators included subjects who did not have asthma. Patients with viral respiratory infections and Mycoplasma pneumoniae, croup, chronic lung disease, and bronchopulmonary dysplasia were enrolled in their study, which could cause different physiopathological processes, for instance, similar abnormalities in proximal and distal airways, resulting in different correlations between $\mathrm{SpO}_{2}$ and PEF. Finally, Connett and Lenney (12) assessed only 26 children with severe acute asthma and obtained negative correlation coefficients, i.e., $r=-0.16$ and -0.10 , with no statistical significance. Because in their study mean predicted PEF value at admission was only $28.3 \%$, its external validity can be questioned, considering that most patients suffering from acute asthma admitted in emergency rooms did not present such low PEF values.

We found patients with high values of PEF and less than $91 \% \mathrm{SpO}_{2}$ and vice versa. These findings conflict with the values proposed by GINA to classify asthma severity and may make some contribution to the understanding of these values in acute asthma. The lack of a higher correlation between $\mathrm{PEF}$ and $\mathrm{SpO}_{2}$ may be ascribed to the type of events assessed by the two methods. Some investigators have suggested that PEF may partially reflect phenomena observed in acute asthma, i.e., only changes observed in the proximal airways $(5,16)$. In contrast, $\mathrm{SpO}_{2}$ may indirectly reflect the ventilation/perfusion mismatch, expressing alterations observed in both proximal and distal airways $(5,16)$. Therefore, the presence of normal PEF values in patients with acute asthma does not exclude the possibility of important abnormalities that may go unnoticed by patients and physicians.

This view is substantiated by the different patterns observed for the two measurements throughout the treatment of acute asthma. Generally, PEF improvement can be more pronounced and faster than $\mathrm{SpO}_{2}$. Considering the physiopathology of acute asthma, the response to treatment in exacerbations seems to have a two-phase course, i.e., a quick initial response followed by a slower one (17-20). The quick phase is probably related to the improvement of smooth muscle contraction (18) and patients in whom this is the major contribution to their disability respond quite well to minimal treatment, even if the obstruction is significant. The slow phase probably depends on the time 
needed to heal the inflammatory process, which has a rather more indolent behavior.

Mihatsch et al. (19), assessing 28 children with acute asthma aged 4 to 14 years, observed that PEF was stabilized $12 \mathrm{~h}$ before $\mathrm{SpO}_{2}$, which was normalized $48 \mathrm{~h}$ later. In addition, Wagner et al. (20) reported a poor association between spirometric alterations and abnormalities in gas exchanges in acute asthma. These investigators stated that the correlation between these parameters tends to improve gradually as the treatment progresses, and concluded that the degree of obstruction in the small airways may differ from those in medium and large airways, explaining the $r$ values detected in the present study.

The moderate correlation between $\mathrm{SpO}_{2}$ and PEF measurements found in the present study supports the view that PEF partially reflects the complex physiopathological findings observed in acute asthma. Therefore, when assessing a patient suffering from an acute attack, the physician should be encouraged to perform both $\mathrm{SpO}_{2}$ and PEF tests in order to define the most adequate treatment.

\section{References}

1. McFadden ER Jr, Kiser R, deGroot WJ. Acute bronchial asthma. Relations between clinical and physiologic manifestations. $N$ Engl J Med 1973; 288: 221-225.

2. Rodrigo G, Rodrigo C. Assessment of the patient with acute asthma in the emergency department. A factor analytic study. Chest 1993; 104: 1325-1328.

3. Bishop J, Carlin J, Nolan T. Evaluation of the properties and reliability of a clinical severity scale for acute asthma in children. J Clin Epidemiol 1992; 45: 71-76.

4. van der Windt DA, Nagelkerke AF, Bouter LM, Dankert-Roelse JE, Veerman AJ. Clinical scores for acute asthma in pre-school children. A review of the literature. J Clin Epidemiol 1994; 47: 635-646.

5. Kerem E, Canny G, Tibshirani R, Reisman J, Bentur L, Schuh S, et al. Clinical-physiologic correlations in acute asthma of childhood. Pediatrics 1991; 87: 481-486.

6. Luaces CC, Garcia Garcia JJ, Garron TP, Sanchez Mora ML, Camacho DE. The usefulness of a clinical scoring system and pulse oximetry $\left(\mathrm{SaO}_{2}\right)$ in assessing the severity of asthmatic crises. An Esp Pediatr 1996; 44: 429-432.

7. Rahnama'i MS, Geilen RP, Singhi S, van den Akker M, Chavannes $\mathrm{NH}$. Which clinical signs and symptoms predict hypoxemia in acute childhood asthma? Indian J Pediatr 2006; 73: 771-775.

8. Boychuk RB, Yamamoto LG, Demesa CJ, Kiyabu KM. Correlation of initial emergency department pulse oximetry values in asthma severity classes (steps) with the risk of hospitalization. Am J Emerg Med 2006; 24: 48-52.

9. Cabral AL, Conceicao GM, Saldiva PH, Martins MA. Effect of asthma severity on symptom perception in childhood asthma. Braz J Med Biol Res 2002; 35: 319-327.

10. National Heart LaBI. Global initiative for asthma. Global strategy for asthma management and prevention. Bethesda: National Institutes of Health; 2002.

11. Godfrey S, Kamburoff PL, Nairn JR. Spirometry, lung volumes and airway resistance in normal children aged 5 to 18 years. $\mathrm{Br} J$ Dis Chest 1970; 64: 15-24.

12. Connett GJ, Lenney W. Use of pulse oximetry in the hospital management of acute asthma in childhood. Pediatr Pulmonol 1993; 15: 345-349.

13. Kano S, Nishima S. Relationship between PEF and $\mathrm{SpO}_{2}$ on acute asthma attack in children. Arerugi 1997; 46: 1265-1272.

14. Yamamoto LG, Wiebe RA, Anaya C, Chang RK, Chang MA, Terada $\mathrm{AM}$, et al. Pulse oximetry and peak flow as indicators of wheezing severity in children and improvement following bronchodilator treatments. Am J Emerg Med 1992; 10: 519-524.

15. Hulley SB, Cummings SR. Designing clinical research. Baltimore: Williams \& Wilkins; 1988.

16. Roca J, Ramis L, Rodriguez-Roisin R, Ballester E, Montserrat JM, Wagner PD. Serial relationships between ventilation-perfusion inequality and spirometry in acute severe asthma requiring hospitalization. Am Rev Respir Dis 1988; 137: 1055-1061.

17. McFadden ER Jr. Clinical physiologic correlates in asthma. J Allergy Clin Immunol 1986; 77: 1-5.

18. Male I, Richter H, Seddon P. Children's perception of breathlessness in acute asthma. Arch Dis Child 2000; 83: 325-329.

19. Mihatsch W, Geelhoed GC, Landau LI, LeSouef PN. Time course of change in oxygen saturation and peak expiratory flow in children admitted to hospital with acute asthma. Thorax 1990; 45: 438-441.

20. Wagner PD, Hedenstierna G, Rodriguez-Roisin R. Gas exchange, expiratory flow obstruction and the clinical spectrum of asthma. Eur Respir J 1996; 9: 1278-1282. 